\title{
Quantitative trait loci and interaction effects responsible for variation in female postmating mortality in Drosophila simulans and $D$. sechellia introgression lines
}

\author{
A Civetta $^{1}, \mathrm{KL}$ Montooth $^{2}$ and M Mendelson ${ }^{1}$ \\ ${ }^{1}$ Department of Biology, University of Winnipeg. Winnipeg, MB, Canada R3B 2E9; ${ }^{2}$ Department of Molecular Biology and Genetics. \\ Cornell University, Ithaca, NY 14853, USA
}

\begin{abstract}
Mating appears to inflict a cost to Drosophila females, resulting in a reduction of their lifespan shortly after mating. Males from different chromosome extracted lines differ significantly in their detrimental effects on postmating female survival, and seminal fluid proteins produced in the male accessory glands are at least partially responsible for the effect. This suggests that there is a genetic basis underlying the male inflicted effect on female's postmating mortality. However, the genes responsible for this effect remain elusive. Using males from introgression lines between $D$. simulans and $D$. sechellia genomes and a quantitative trait locus (QTL) mapping approach, we identified chromosomal regions that affect postmating mortality of females. We found
\end{abstract}

a second chromosome QTL with an effect on average female lifespan after mating and a third chromosome QTL with an effect on postmating female mortality rate. Under the general observation of a faster divergence of sex-related genes among closely related species, it is predicted that genes for reproductive traits other than hybrid sterility will show evidence of epistatic effects when brought into a heterospecific background. We detected a significant epistatic genetic effect on postmating female mortality rate that supports this prediction.

Heredity (2005) 94, 94-100. doi:10.1038/sj.hdy.6800570

Published online 8 September 2004

Keywords: QTL mapping; postmating mortality; Drosophila; interspecies introgressions; sex-traits evolution; speciation

\section{Introduction}

Several studies have brought attention to the adverse effects that mating can have on females. In Drosophila melanogaster, when females mate throughout their lifespan, a harm inflicted by males is seen as a reduction of the female's lifespan shortly after mating (Fowler and Partridge, 1989; Chapman et al, 1993, 1995). There is indication that female lifespan reduction after mating has a genetic basis, as females mated to males from different chromosome extracted lines experience significant differences in postmating survival (Civetta and Clark, 2000; Sawby and Hughes, 2001). Moreover, several independent studies suggest that accessory gland proteins transferred in the male ejaculate are potentially responsible for reduced female lifespan. Females mated to mutant $D$. melanogaster males deficient in accessory gland main cell products did not experience the reduction in survival seen in females mated to control males, suggesting that the transfer of accessory gland secretions underlies the costly effect of mating (Chapman et al, 1995).

Specific proteins produced in the male accessory glands stimulate different postmating responses in

Correspondence: A Civetta, Department of Biology, University of Winnipeg. 515 Portage Avenue, Winnipeg, MB, Canada R3B 2E9.

E-mail:a.civetta@uwinnipeg.ca

Received 2 March 2004; accepted 7 July 2004; published online 8 September 2004 females. Accessory gland proteins (Acps) are responsible for increased ovulation and egg-laying rate, efficient sperm storage, decreased receptivity for further matings and better sperm competitive abilities (Wolfner, 2002). Acp $62 F$ has been shown to affect adult female lifespan when it is repeatedly overexpressed in D. melanogaster (Lung et al, 2002), pointing at the potential role of Acp62F in reducing female lifespan after mating. However, we do not have evidence that natural variation in a male expressed gene product, such as Acp62F, transferred to the female during mating contributes to the observed decrease in female lifespan after mating. We also do not know whether such postmating mortality effect occurs in other species of Drosophila.

An attempt to map genes responsible for the postmating survival cost inflicted by males on females could use different approaches. One possibility is to select for Drosophila strains with males that inflict either low or high cost on females after mating and use such strains to map genetic differences. An alternative approach is to take advantage of differences fixed among populations or species. Divergence between species is usually at its maximum for precopulatory and postcopulatory reproductive traits (Eberhard, 1985; Pitnick and Markow, 1994; Arnqvist, 1998; Civetta and Singh, 1998; Pitnick et al, 1999; Arnqvist and Rowe, 2002). This suggests that interspecific replacement of genomic regions can be used to evaluate the effect of such replacements on traits of reproduction without the need of selecting a priori for extreme 
phenotypes. However, genetic introgressions among many species are limited by the sterility and/or inviability of their hybrid progeny. Species of the simulans clade (D. simulans, D. mauritiana and D. sechellia) are an exception as they can be crossed to produce viable and fertile hybrid females that can be backcrossed to parental species to randomly generate Drosophila strains with interspecific genomic substitutions.

Interspecies introgressed lines have part of a species genome substituted for the genome of a closely related species and they are, at least for some genes, similar to interspecies hybrids in the sense that heterospecific gene interactions are possible. Studies of the genetics of interspecies hybrid sterility have shown that candidate genes have a stronger effect in the presence of heterospecific or even conspecific epistatic genes (Palopoli and $\mathrm{Wu}, 1994$; Perez and $\mathrm{Wu}, 1995$; Orr, 1997; Orr and Presgraves, 2000; Turelli and Orr, 2000; Tao et al, 2003). Conspecific epistatic effects in a heterospecific background were clearly demonstrated in interspecies introgression studies used to map Odysseus, a gene responsible for hybrid male sterility with a complete sterility effect only when cointrogressed with other conspecific genes (Perez and Wu, 1995). There is also good evidence that male expressed genes show patterns of rapid divergence between species (Civetta and Singh, 1999; Singh and Kulathinal, 2000; Swanson and Vacquier, 2002). Under this pattern of fast sex-related genes evolution, it is possible that the type of epistatic effects observed for the sterility phenotype among closely related species could be a property shared by sex-related traits in general and not unique to sterility.

Previous studies on the effect of mating on female survival have shown that average lifespan is not necessarily lower among mated than virgin flies (Civetta and Clark 2000; Carey et al, 2002). However, postcopulatory survival cost effects seems to be stronger among young females indicating an earlier rather than later age effect. Owing to the early age effect of mating on female survival and the large number of flies to be tested for QTL mapping purposes, we used single rather than multiple mating of $D$. simulans females to $D$. simulans males with randomly introgressed $D$. sechellia chromosomal fragments (IG lines). A QTL approach is used to identify introgressed genomic regions that are associated with female postcopulatory lifespan. We find that (1) single candidate loci and epistatic interactions contribute to the male adverse effects on female survival after mating, (2) a QTL with single and complex genetic effects on postmating female mortality rate maps close to a male accessory gland gene previously suggested to have toxic effects when over expressed and (3) the type of epistatic interaction found for introgressed simulans/sechellia male's postcopulatory survival cost inflicted on $D$. simulans females mimics results found for hybrid male sterility.

\section{Materials and methods}

\section{Drosophila stocks and maintenance}

A total of 101 introgressed recombinant inbred lines (IG lines) were used in this study. The lines were a result of genetic introgression between $D$. simulans from the 'sim2CA' line (Winters, CA, USA) and the closely related D. sechellia (Drosophila Stock Species Centre, 14021-
0248.4). D. simulans females were crossed with $D$. sechellia males and the resulting $F_{1}$ females were backcrossed to $D$. simulans males. The subsequent $\mathrm{F}_{2}$ males were backcrossed to $D$. simulans females. The $\mathrm{F}_{3}$ progeny were separated into lines that were made homozygous by 14 generations of single-pair sibling mating (Dermitzakis et al, 2000; Civetta et al, 2002). Every generation of sibmating causes a nearly $20 \%$ loss in heterozygosity (Hedrick, 1983, pp 98-100) so that after 14 generations less than $1 \%$ heterozygosity might still segregate among the IG lines.

All fly stocks were maintained on a $12 \mathrm{~h}$ light/dark cycle at $24^{\circ} \mathrm{C}$. Stocks were kept in bottles containing approximately $50 \mathrm{ml}$ of cornmeal-molasses media.

\section{Mortality assay}

Virgin D. simulans 'sim2CA' females were collected at eclosion using $\mathrm{CO}_{2}$ anaesthesia and kept in groups of five in individual shell vials containing cornmealmolasses media. After 4-6 days, the medium was examined for eggs and larvae to ensure that females were virgins. $D$. simulans females were aspirated into individual vials along with 1 or 2 virgin 4-6-day-old males from an IG line. For each IG line, we set up at least 10 mating vials. Vials were checked every $10 \mathrm{~min}$ and males were removed after a single mating. Each IG line was tested again on a different day. We tested an average of 18 males per IG line.

Female mortality was scored every day until all females had died. Females were transferred to fresh vials every 4 days. For the first 8 days, each female was kept in her own vial. After 8 days, all females mated to the same IG males were kept together in a single vial to a limit of 10 females per vial. The assumption is made here that batches of food made and used at each time of transfer are quite homogeneous so that females mated to different IG males share very common environments. The number of progeny produced by each singly mated female was counted over the first two vials (8 days in isolation). Females that produced fewer than 20 progeny flies were removed from the experiment as these might include females that mate for a very short period of time, males that produce low numbers of sperm, low quality food media or possible injury/ infection affecting either egg-laying or progeny viability (Civetta et al, 2002).

For each group of females mated to males from an IG line, average lifespan and mortality rate were recorded as described by Civetta and Clark (2000). Briefly, average lifespan is the average age at death, and the mortality rate is calculated as a function of the number of females alive each day relative to the previous day. A linear relationship can be obtained for the mortality rate as a function of age at death by fitting the logarithm of the mortality rate to the squared age at death. The intercept as well as the average slope of such function can be used as indicators of changes in the mortality rate after mating. This is because a lower slope results in a higher intercept, reflecting higher mortality shortly after mating. We used both parameters of the mortality function as indicators of the cost inflicted by IG males on $D$. simulans females after mating. The genetic component of the phenotypic variation among IG lines was tested using analysis of variance with genetic line and trial nested 
within line as factors. All statistical analyses were carried out using SPSS version 10.1.

\section{QTL mapping}

To establish possible gene-trait associations, we combined information on the phenotypic scores obtained from different IG lines with information on the genetic makeup of IG lines for 29 different molecular markers spread across the three major chromosomes of Drosophila. Of the 29 molecular markers, information on 27 markers has already been published (Civetta et al, 2002; Civetta and Cantor, 2003). The two new molecular markers added to the map are DMMP20 (2-133.8) and rhob (3-5). PCR amplification conditions were 45 cycles of $95^{\circ} \mathrm{C}$ for $1 \mathrm{~min}, 55^{\circ} \mathrm{C}$ for $1 \mathrm{~min}$ and $72^{\circ} \mathrm{C}$ for $1.5 \mathrm{~min}$ followed by $72^{\circ} \mathrm{C}$ extension for $2 \mathrm{~min}$. The oligonucleotide primers used to amplify DMMP20 and rhob are described elsewhere (Goldstein and Clark, 1995; Colson and Goldstein, 1999).

QTL mapping analysis was carried out using QTL Cartographer version 2.0 (Wang et al, 2003). For each trait, we explored single marker-trait associations using linear regression analysis. We tested for the presence of significant QTLs between markers using interval and composite interval mapping with $5 \mathrm{cM}$ intervals. In interval mapping (IM), genotypes at positions between flanking markers are imputed and then tested for the null hypothesis of no genetic effect of a given position on the quantitative trait being measured (Lander and Botstein, 1989). Composite interval mapping (CIM) is an interval mapping approach that conditions the presence of a QTL on background genetic markers (Jansen and Stam, 1994; Zeng, 1994). We chose background markers using a forward regression approach with background elimination and an exclusion window of $50 \mathrm{cM}$ flanking the test position. The significance of any potential QTL was calculated by comparing the likelihood scores for any given QTL to the distribution of likelihood scores obtained from 1000 random permutations of the trait data across the marker genotype data (Churchill and Doerge, 1994). The distribution of likelihood scores from the permutation analysis allowed us to obtain critical threshold levels for the test statistic that control the experiment wise type I error rates for the large number of multiple tests inherent in QTL mapping.

We tested all possible two-locus models, including genetic interactions, for effects on the phenotypes using the statistical framework of Sen and Churchill (2001). This approach is a type of Bayesian interval mapping that imputes multiple genotype data sets from the observed marker genotypes and map. These genotype data sets are used in combination with the observed phenotype data to generate approximations to the posterior distributions of QTL location and effect. We imputed 100 pseudomarker data sets at a spacing of $5 \mathrm{cM}$. We scanned for significant two-locus models using experiment wise critical threshold values obtained from 300 permutations.

\section{Results}

\section{Differences in mortality among females}

The average lifespan of females mated to males from 101 different IG lines varied significantly among lines

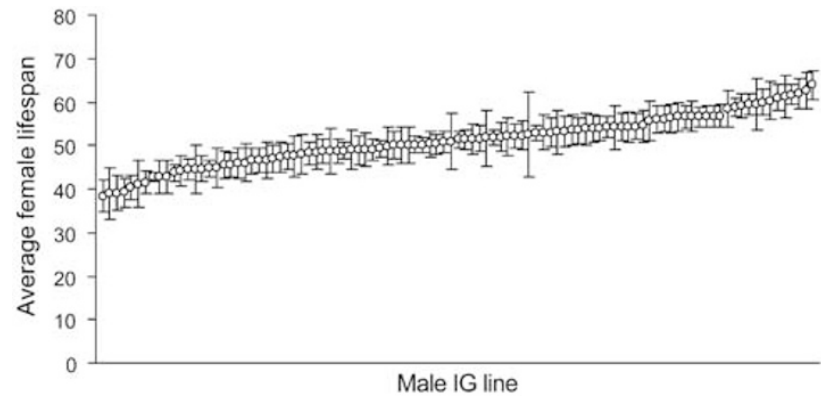

Figure 1 Variation in average D. simulans female lifespan (in days) after mating to males from different IG lines. The average lifespan of females mated to each IG line is plotted in rank order with associated standard errors.

$\left(\mathrm{F}_{100,1633}=2.89 ; P<0.001\right)$ (Figure 1$)$, and the effect remained significant when tested against trials as the error mean square $\left(\mathrm{F}_{100,106}=1.56 ; P=0.012\right)$. This suggests that although part of the differences between females mated to different IG males could be partially environmental (ie a trial effect), there remains a detectable and significant difference between IG lines with different genetic makeup even when tested against error due to replicate trials. The number of progeny produced by females was not correlated with average postmating mortality $(r=-0.01 ; P=0.925 ; N=95)$, mortality rate measured as the slope of mortality function ( $r=-0.097 ; P=0.350 ; N=95)$ or early mortality measured as the intercept of the mortality function $(r=0.100$; $P=0.337 ; N=94)$. We found age-specific differences in survival among females mated to males from different IG lines (Figure 2a) with the average lifespan of mated females being significantly correlated to both early mortality $(r=-0.426, P<0.0001, N=97)$ and mortality rate (slope of mortality function; $r=-0.245, P=0.015$, $N=99$ ). There is also a significant and negative correlation between early postmating mortality and postmating mortality rate $(r=-0.718, P<0.0001, N=97)$.

It appears that single mating does not necessarily lead to postmating mortality differences between mated and virgin females. In fact, females mated to some IG lines lived longer than virgin females (Figure 2a). The costly effect of single mating impacts early mortality, with mortality rates becoming more homogenous between virgin and mated females at later ages. This is seen in Figures $2 a$ and $b$, where the mortality data points of females appear less intermixed among virgins and mated flies at younger ages. This result is consistent with prior studies that suggested a stronger costly effect of mating on survival among young rather than old females (Civetta and Clark 2000; Carey et al, 2002). D. simulans females mated to males from the same species show higher mortality shortly after mating than $D$. simulans females mated to $D$. sechellia males. However, the postmating mortality rates are similar (Figure $2 \mathrm{~b}$ and c). Females mated to different IG lines exhibited a range of effects on postmating mortality, with some IG lines decreasing and other IG lines increasing mortality relative to females mated to either parental species (Figure 2c). Phenotypic transgressions over parental values are not uncommon among interspecific hybrids (Dobzhansky, 1952; Civetta and Singh, 1998) and such 

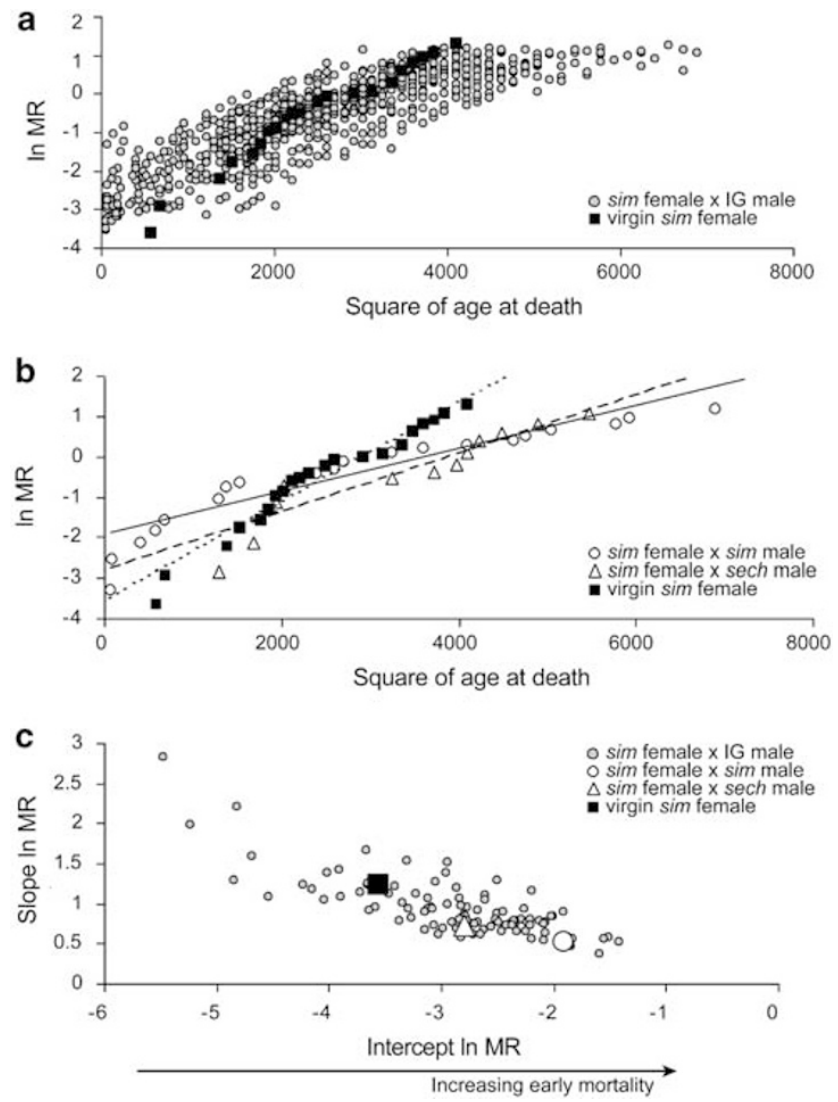

Figure 2 (a) Mortality rate as a function of the square of age at death of virgin $D$. simulans females and $D$. simulans females mated to IG males. (b) Mortality rate of virgin $D$. simulans females and $D$. simulans females mated to $D$. simulans or $D$. sechellia males. Solid and dashed lines are linear functions fitted to females mated to $D$. simulans and D. sechellia, respectively, while the dotted line is for virgins. (c) Average of the slope $\left(\times 10^{3}\right)$ of the mortality function of virgin $D$. simulans females and those mated to different males versus the intercept of the mortality function.

transgressions are also seen in QTL studies of trait variation using recombinant inbred lines of $D$. melanogater (Nuzhdin et al, 1997; Gurganus et al, 1998; Gleason et al, 2002).

\section{Single-locus effects and two-loci interactions}

Mapping at molecular markers using linear regression reveals a significant association between variation in average postmating female lifespan and a second chromosome molecular marker (ninaC) at cytological position 27F3 $\left(\mathrm{F}_{1,99}=11.95 ; P=0.0007<\right.$ Bonferroni corrected $\left.P^{\prime}=0.025 / 29\right)$. Significant associations with markers rhob (62A1-2) and ple (65C3) on the third chromosome were found for different aspects of female postmating mortality (ie average survival, intercept and slope of MR function), but none of the associations were significant after Bonferroni correction.

A single QTL for average female lifespan after mating was detected on the second chromosome $(\mathrm{LR}=12.03$, $P<0.01$, IM; LR=9.33, $P<0.10, \mathrm{CIM}$ ) (Figure 3). The location of this QTL is in agreement with the significant effect detected at molecular marker ninaC (27F3) using single marker-trait regression analysis. A D. sechellia

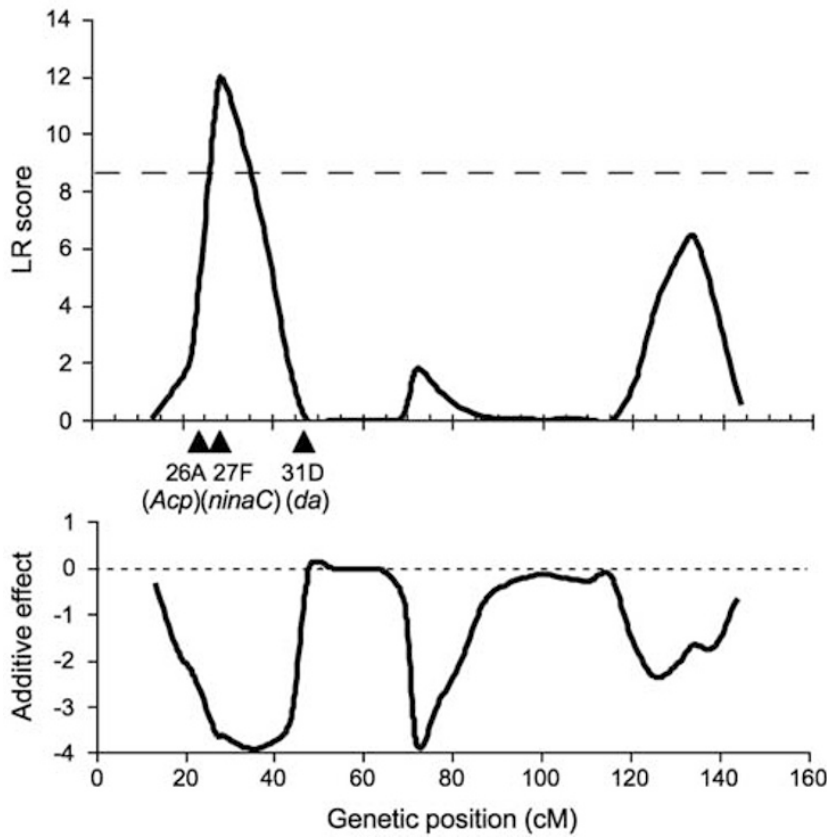

Figure 3 Second chromosome QTL for differences in D. simulans female average lifespan after mating to males from different IG lines. Likelihood ratio (LR) values are from IM. The experiment wise significance level $(P<0.05)$ is shown as a dashed line across genetic positions. Genetic positions are represented as units of recombination (cM) across the chromosome. The name and cytological position (see FlyBase: http://flybase.bio.indiana.edu/) of molecular markers at or flanking the QTL are shown. The additive effect reflects the effect of substituting a $D$. sechellia allele at each genetic position, with a zero value (dotted line) denoting no phenotypic difference between alleles.

allele introgression at marker ninaC inflicts a greater cost to females, as it causes a reduction in females' average postmating lifespan from $52 \pm 0.6$ to $44 \pm 1.8$ days.

Two significant QTL explaining variation in the slope of the mortality function of females after mating were found on the third chromosome at marker rhob (62A1-2; $\mathrm{LR}=10.82, P<0.02, \mathrm{CIM})$ and between markers $p n t$ and anp (94E11-99E2; LR =9.12, $P<0.05, C I M)$ when using composite interval mapping (Figure 4). However, the same QTL were not significant when an interval mapping approach was used. This is likely because the two QTL on chromosome 3 have genetic effects in the opposite direction (Figure 4) and there appear to be epistatic interactions, as well (see below). Since IM does not account for these types of genetic background effects, a CIM approach may better model the data (Montooth et al, 2003). IG males carrying a D. sechellia allele at marker rhob cause a significant reduction in the average slope of the mortality function for females $\left(F_{1,83}=5.11\right.$; $P=0.026)$, indicating increased early mortality. Interestingly, this mapped QTL includes Acp62F, an accessory gland protein that significantly reduces survival when it is overexpressed in Drosophila melanogaster (Lung et al, 2002).

We identified a two-locus model underlying variation in the slope of the mortality function that includes the third chromosome QTL at rhob and its interaction with a region on the $X$ chromosome near the marker Passover (19E3). The full model, including additive effects of the 

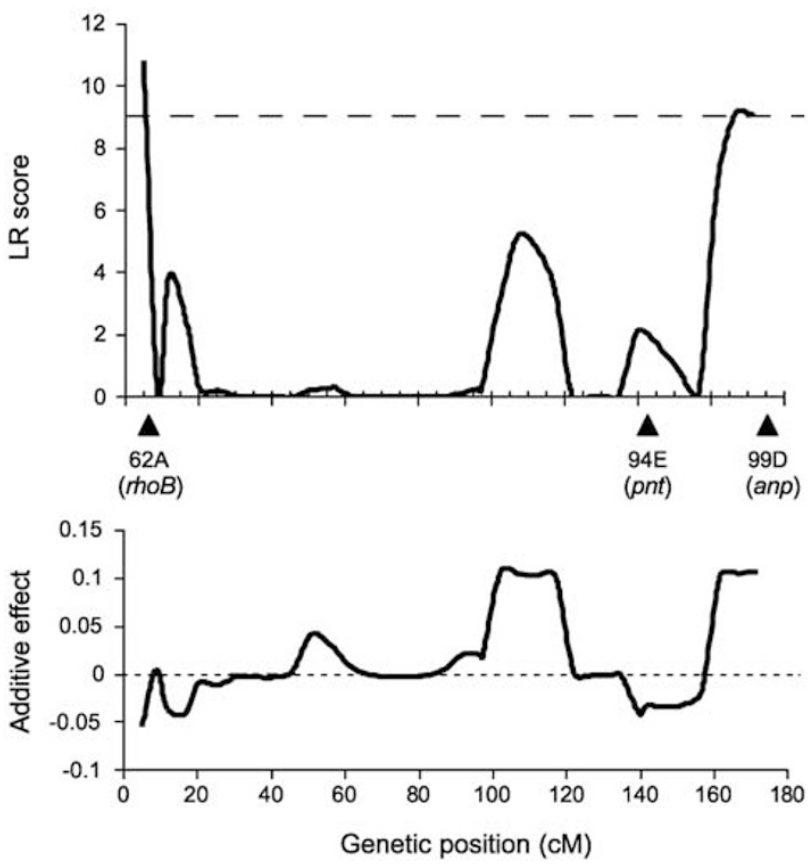

Figure 4 Third chromosome QTL for differences in $D$. simulans female mortality rate after mating to males from different IG lines. LR values are from CIM. The map positions, significance levels and additive effects are shown as in Figure 3.

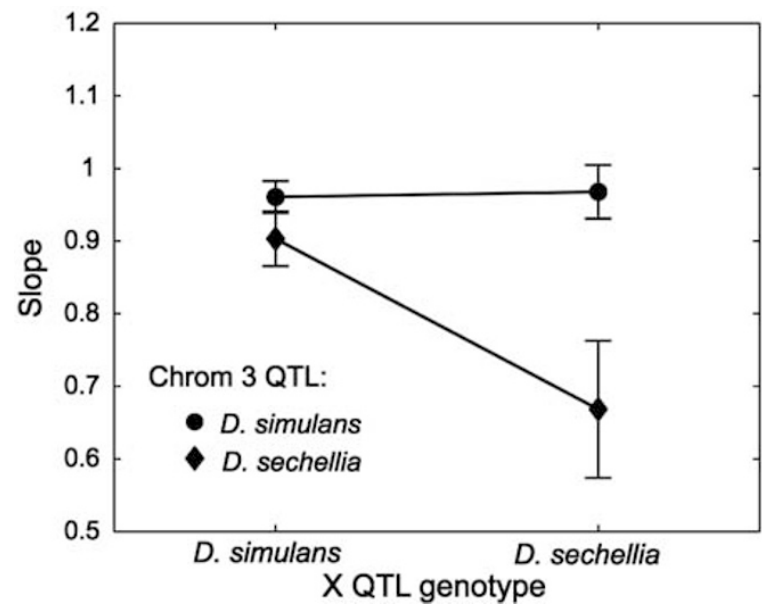

Figure 5 Interaction between the third chromosome QTL at rhob and a region of the $X$ chromosome. Plotted are weighted means and variances of the slope of the mortality function $\left(\times 10^{3}\right)$ for the four two-locus genotypes from the 100 imputed genotype data sets (Sen and Churchill 2001).

two loci and their interaction, was significant at the experiment wise $10 \%$ error rate $\left(\mathrm{LOD}_{\text {full }}=4.22\right)$. The difference between the LOD scores for the full model and a reduced model containing only additive effects gave a LOD score of 2.36 for the interaction effect. The nominal $P$-value associated with this LOD score was less than 0.001 . Males carrying the $D$. sechellia genotype at both genomic positions confer a greater cost to females, as seen by the decrease in the slope of the mortality function of females mated to these males (Figure 5).

\section{Discussion}

Selection experiments reveal that males inflict a higher postmating survival cost to females when the two sexes are not allowed to coevolve (Rice, 1996). Thus, heterospecific males would also be expected to cause higher cost unless the costly effect of mating is lost in males that have diverged from $D$. simulans females for a long time (ie D. sechellia males). This study supports the latter prediction, as we observed a higher postmating female mortality cost shortly after mating to conspecific males than to heterospecific males.

Although the postmating mortality cost effect was overall higher for females mated to conspecific males, we found that $D$. sechellia introgressions into several regions of the $D$. simulans genomic background increased the cost of mating to females. IG males carrying a $D$. sechellia allele at the second chromosome QTL caused a reduction in average female survival after mating, and a D. sechellia allele near rhob on the third chromosome resulted in higher female mortality shortly after mating. However, we also found a third chromosome QTL near markers pnt and anp where the introgression of a $D$. sechellia allele resulted in lower postmating female mortality cost. The lack of directionality on the phenotypic effect of $D$. sechellia allele introgression in a $D$. simulans background is in agreement with observations relating to other phenotypes analyzed among this set of introgressed lines (Dermitzakis et al, 2000; Civetta et al, 2002; Civetta and Cantor, 2003) and is suggestive of a polygenic basis of inheritance involving interactions rather than simple additive effects.

Interspecific hybridizations among closely related species of Drosophila result in hybrid male sterility due to the cumulative effect of several genes with epistatic interactions (Dobzhansky, 1937; Muller, 1940, 1942; Presgraves, 2003). We detected epistatic interactions underlying the postmating mortality cost to females. The QTL underlying the slope of the mortality function near rhob on the third chromosome interacted with a region of the $X$ chromosome. Introgression of the $D$. sechellia genome at this region of the $X$ had no effect on cost when the genetic background at the third chromosome QTL was from the D. simulans genome. However, when the genetic background was also from $D$. sechellia, there was a dramatic increase in the cost to females, manifested as an increase in mortality rates postmating.

The detrimental effect of epistatic interactions among conspecific genes has been shown for hybrid incompatibilities when such epistatic alleles are moved into a heterospecific genetic background (Cabot et al, 1994; Palopoli and Wu, 1994; Orr, 1995; Orr and Irving, 2001; Tao et al, 2003). Studies of hybrid male sterility among different Drosophila species show that sterility factors have the strongest effect when cointrogressed with conspecific genes (Naveira and Fontdevila, 1986; Perez and $W u, 1995)$. A similar situation is seen here where the introgression of a sechellia allele at a third chromosome locus has a significant effect on postmating mortality cost only when cointrogressed with an $X$ chromosome sechellia allele.

The faster evolution of male reproductive genes compared to genes unrelated to sexual reproduction (Civetta and Singh, 1999; Singh and Kulathinal, 2000; Swanson and Vacquier, 2002) suggests that hybrid male 
sterility could be only one of several reproductive traits likely to suffer hybrid epistatic incompatibilities due to rapid accumulation of genetic differences between closely related species at genes that control such traits. Prior studies have not been conclusive about epistatic effects underlying species diversification (Orr, 2001). This may be a consequence of the inherent difficulties in detecting genetic interactions (Mackay, 2001). The detection of a significant gene interaction effect underlying differences in postmating male inflicted mortality cost among IG lines is suggestive of the idea that faster divergence of sex-related genes between closely related species leads to interspecies gene interaction effects in sexual traits other than sterility. We have also detected a significant $\mathrm{X}$-autosome interaction for another male reproductive trait in the simulans/sechellia IG lines using sperm competitive scores published elsewhere (Civetta et al, 2002).

Postmating female mortality could result from any number of sex-related phenomena, such as male aggression and/or the amount of seminal fluid transferred to the female. It is interesting to note that Acp62F maps to the same genomic region as the third chromosome QTL underlying increased cost. Acp62F encodes a male accessory gland protein shown to be a trypsin inhibitor with a toxic effect when ectopically expressed in transgenic flies (Lung et al, 2002). However, the region mapped on the third chromosome is clearly not the only gene region influencing female survival after mating. We find evidence that a complex genetic architecture involving genetic factors from different chromosomes with potential interactions underlies postmating cost to female Drosophila. The role of genetic interactions in explaining phenotypic variation in a complex trait such as postmating mortality rate points at the need to further investigate through full genome scans beyond singlelocus effects.

\section{Acknowledgements}

We thank A Baljic and N Bansal who helped us set up the mortality assays and J Fisher who contributed with the development and scoring of molecular markers. We greatly appreciate comments received from AG Clark, RS Singh and JR Walters. This work was funded by a grant from the Natural Sciences and Engineering Research Council of Canada to A Civetta.

\section{References}

Arnqvist G (1998). Comparative evidence for the evolution of genitalia by sexual selection. Nature 393: 784-786.

Arnqvist G, Rowe L (2002). Correlated evolution of male and female morphologles in water striders. Evolution 56: 936-947.

Cabot EL, Davis AW, Johnson NA, Wu CI (1994). Genetics of reproductive isolation in the Drosophila simulans clade: complex epistasis underlying hybrid male sterility. Genetics 137: 175-189.

Carey JR, Liedo P, Harshman L, Zhang Y, Muller H-G, Partridge L, Wang J-L (2002). A mortality cost of virginity at older ages in female Mediterranean fruit flies. Exp Gerontol 37: 507-512.

Chapman T, Hutchings J, Partridge L (1993). No reduction in the cost of mating for Drosophila melanogaster females mating with spermless males. Proc R Soc Lond B Biol Sci 253: 211-217.
Chapman T, Liddle LF, Kalb JM, Wolfner MF, Partridge L (1995). Cost of mating in Drosophila melanogaster females is mediated by male accessory gland products. Nature 373: 241-244.

Churchill GA, Doerge RW (1994). Empirical threshold values for quantitative trait mapping. Genetics 138: 963-971.

Civetta A, Cantor EJF (2003). The genetics of mating recognition between Drosophila simulans and D. sechellia. Genet Res $\mathbf{8 2}$ 117-126.

Civetta A, Clark AG (2000). Correlated effects of sperm competition and postmating female mortality. Proc Natl Acad Sci USA 97: 13162-13165.

Civetta A, Singh RS (1998). Sex and speciation: genetic architecture and evolutionary potential of sexual versus nonsexual traits in the sibling species of the Drosophila melanogaster complex. Evolution 52: 1080-1092.

Civetta A, Singh RS (1999). Broad-sense sexual selection, sex gene pool evolution, and speciation. Genome 42: 1033-1041.

Civetta A, Waldrip-Dail HM, Clark AG (2002). An introgression approach to mapping differences in mating success and sperm competitive ability in Drosophila simulans and $D$. sechellia. Genet Res 79: 65-74.

Colson I, Goldstein DB (1999). Evidence for complex mutations at microsatellite loci in Drosophila. Genetics 152: 617-627.

Dermitzakis ET, Masly JP, Waldrip HM, Clark AG (2000). NonMendelian segregation of sex chromosomes in heterospecific Drosophila males. Genetics 154: 687-694.

Dobzhansky Th (1937). Genetics and the Origin of Species, Columbia University Press: New York.

Dobzhansky Th (1952). Nature and origin of heterosis. In: Gowen JW (ed) Heterosis, Iowa State College Press: Ames, IA. pp 218-223.

Eberhard WG (1985). Sexual Selection and Animal Genitalia, Harvard University Press: Cambridge, MA.

Fowler K, Partridge L (1989). A cost of mating in female fruitflies. Nature 338: 760-761.

Gleason JM, Nuzhdin SV, Ritchie MG (2002). Quantitative trait loci affecting a courtship signal in Drosophila melanogaster. Heredity 89: 1-6.

Goldstein DB, Clark AG (1995). Microsatellite variation in North American populations of Drosophila melanogaster. Nucleic Acids Res 23: 3882-3886.

Gurganus MC, Fry JD, Nuzhdin SV, Pasyukova EG, Lyman RF, Mackay TF (1998). Genotype-environment interaction at quantitative trait loci affecting sensory bristle number in Drosophila melanogaster. Genetics 149: 1883-1898.

Hedrick PW (1983). Genetics of Populations, Science Books International: Boston. pp 98-100.

Jansen RC, Stam P (1994). High resolution of quantitative traits into multiple loci via interval mapping. Genetics $\mathbf{1 3 6}$ 1447-1455.

Lander ES, Botstein D (1989). Mapping Mendelian factors underlying quantitative traits using RFLP linkage maps. Genetics 121: 185-199.

Lung O, Tram U, Finnerty CM, Eipper-Mains MA, Kalb JM, Wolfner MF (2002). The Drosophila melanogaster seminal fluid protein Acp62F is a protease inhibitor that is toxic upon ectopic expression. Genetics 160: 211-224.

Mackay TF (2001). The genetic architecture of quantitative traits. Ann Rev Genet 35: 303-339.

Montooth KL, Marden JH, Clark AG (2003). Mapping determinants of variation in energy metabolism, respiration and flight in Drosophila. Genetics 165: 623-635.

Muller HJ (1940). Bearing the Drosophila work on systematics. In: Huxley JS (ed) The New Systematics, Clarendon Press: Oxford. pp 185-268.

Muller HJ (1942). Isolating mechanisms, evolution, and temperature. Biol Symp 6: 71-125.

Naveira H, Fontdevila A (1986). The evolutionary history of Drosophila buzzatii. XII. The genetic basis of sterility factors in hybrids between $D$. buzzatii and its sibling $D$. serido from Argentina. Genetics 144: 841-857. 
Nuzhdin SV, Pasyukova EG, Dilda CL, Zeng ZB, Mackay TF (1997). Sex-specific quantitative trait loci affecting longevity in Drosophila melanogaster. Proc Natl Acad Sci USA 94: 9734-9739.

Orr HA (1995). The population genetics of speciation: the evolution of hybrid incompatibilities. Genetics 139: 1805-1813.

Orr HA (1997). Haldane's rule. Ann Rev Ecol Syst 28: 195-218.

Orr HA (2001). The genetics of species differences. Trends Ecol Evol 16: 343-350.

Orr HA, Irving S (2001). Complex epistasis and the genetic basis of hybrid sterility in the Drosophila pseudoobscura Bogota-USA hybridization. Genetics 158: 1089-1100.

Orr HA, Presgraves DC (2000). Speciation by postzygotic isolation: forces, genes and molecules. BioEssays 22: 1085-1094.

Palopoli MF, Wu CI (1994). Genetics of hybrid male sterility between drosophila sibling species: a complex web of epistasis is revealed in interspecific studies. Genetics 138: 329-341.

Perez DP, Wu CI (1995). Further characterization of the hybrid sterility gene, Odysseus (Ods), in the Drosophila simulans clade: one gene is not enough. Genetics 140: 201-206.

Pitnick S, Markow TA (1994). Large-male advantages associated with costs of sperm production in Drosophila hydei, a species with giant sperm. Proc Natl Acad Sci USA 91: 9277-9281.

Pitnick S, Markow TA, Spicer GS (1999). Evolution of multiple kinds of female sperm-storage organs in Drosophila. Evolution 53: 1804-1822.
Presgraves DC (2003). A fine-scale genetic analysis of hybrid incompatibilities in Drosophila. Genetics 163: 955-972.

Rice WR (1996). Sexually antagonistic male adaptation triggered by experimental arrest of female evolution. Nature 381: 232-234.

Sawby R, Hughes KA (2001). Male genotype affects female longevity in Drosophila melanogaster. Evolution 55: 834-839.

Sen S, Churchill GA (2001). A statistical framework for quantitative trait mapping. Genetics 159: 371-387.

Singh RS, Kulathinal RJ (2000). Sex gene pool evolution and speciation: a new paradigm. Genes Genet Syst 75: 119-130.

Swanson WJ, Vacquier VD (2002). The rapid evolution of reproductive proteins. Nat Rev Genet 3: 137-144.

Tao Y, Zeng ZB, Li J, Hartl DL, Laurie CC (2003). Genetic dissection of hybrid incompatibilities between Drosophila simulans and D. mauritiana. II. Mapping hybrid male sterility loci on the third chromosome. Genetics 164: 1399-1418.

Turelli M, Orr HA (2000). Dominance, epistasis and the genetics of postzygotic isolation. Genetics 154: 1663-1679.

Wang S, Basten CJ, Zeng ZB (2003). QTL Cartographer, Version 2.0, Department of Statistics, North Carolina State University: Raleigh, NC.

Wolfner MF (2002). The gifts that keep on giving: physiological functions and evolutionary dynamics of male seminal proteins in Drosophila. Heredity 88: 85-93.

Zeng Z-B (1994). Precision mapping of quantitative trait loci. Genetics 136: 1457-1468. 